\title{
Spontaneous Basilar-Membrane Oscillation (SBMO) and Coherent Reflection
}

\author{
Egbert de Boer ${ }^{1}$ and Alfred L. Nuttall ${ }^{2,3}$ \\ ${ }^{1}$ Academic Medical Center, University of Amsterdam, Room D2-226, Meibergdreef 9, 1105 AZ, Amsterdam, The Netherlands \\ ${ }^{2}$ Oregon Hearing Research Center, NRC04, Oregon Health \& Science University, 3181 SW Sam Jackson Park Road, \\ Portland, OR 97239-3098, USA \\ ${ }^{3}$ Kresge Hearing Research Institute, University of Michigan, 1301 E. Ann Street, Ann Arbor, MI 48109-0506, USA
}

Received: 16 August 2005; Accepted: 19 October 2005; Online publication: 21 January 2006

\begin{abstract}
In a previous report (in JARO) we have described a relatively high-frequency $(15 \mathrm{kHz})$ spontaneous oscillation of the basilar membrane (SBMO) in a guinea pig ear; this oscillation was accompanied by a spontaneous otoacoustic emission (SOAE) at the same frequency. During the spontaneous oscillation and after it had subsided, the mechanical frequency response of the basilar membrane was measured by way of a wide-band random-noise stimulus, and it showed a number of spectral peaks, one of which having the frequency of the original oscillation. This pattern of peaks cannot be explained by assuming a single place of reflection in the cochlea. In this paper the process of 'coherent reflection' is artificially evoked in a three-dimensional model of the cochlea by imposing random place-fixed irregularities to the basilar-membrane impedance. It is shown that in the model a series of peaks arises in the frequency spectrum of the basilar-membrane response which phenomenon resembles the one found in the experimental animal. It is also shown that these peaks are actually due to superposition of the primary wave and a wave resulting from 'coherent reflection' which is reflected at the stapes. When the intensity of the acoustic stimulus signal is increased, the relative sizes of these peaks in the simulation diminish in about the same way as in the experiment.
\end{abstract}

Correspondence to: Alfred L. Nuttall - Oregon Hearing Research Center, NRC04 - Oregon Health \& Science University · 3181 SW Sam Jackson Park Road, Portland, OR 97239-3098, USA. Telephone: +1-503-4948032; fax: +1-503-4945656; email: nuttal@ohsu. edu
It is concluded that coherent reflection most likely is the cause of the 'extra peaks', and that this concept can also explain the observed level dependence of these peaks. The findings of this study lead to a minor refinement regarding the actual requirements for coherent reflection to arise.

Keywords: active cochlea, basilar membrane, coherent reflection, spontaneous oscillation

\section{INTRODUCTION}

In a previous report we have described a relatively high-frequency $(15 \mathrm{kHz})$ spontaneous basilar-membrane oscillation in a guinea pig ear (SBMO), which was accompanied by a spontaneous otoacoustic emission (SOAE) at the same frequency (Nuttall et al. 2004). Spontaneous otoacoustic emissions are sounds that are emitted from a human or animal ear, and are commonly considered to originate in mammals from oscillations of the organ of Corti, in particular, the basilar membrane (BM). The aforementioned observation of a spontaneous BM oscillation (SBMO) and a coincident spontaneous otoacoustic emission (SOAE) with the same frequency considerably strengthens this opinion. It has been proposed that in mammals SOAEs are due to longitudinal inhomogeneities of the organ of Corti, which cause multiple internal reflections in the cochlea (Kemp 1978, 1979; Shera 2003). The same explanation should be true for spontaneous oscillations of the basilar membrane. The inhomogeneities may be due to morphological variations such as 
the presence or absence of outer hair cells (OHCs), see Lonsbury-Martin et al. (1988) and Hilger et al. (1995), or result from more subtle variations in mechano-electric properties of individual cells (in particular, OHCs). The commonly accepted mechanism of multiple reflection involves two sub-processes:

I. Reflection of the forward-traveling wave, and

II. Reflection of the backward-traveling (retrograde) wave by the stapes.

If the wave reflected in stage II enhances the primary wave, the possibility of spontaneous oscillation of the $\mathrm{BM}$ is increased. This enhancement can occur at a limited number of frequencies, which explains that SOAEs often occur in groups with a specific frequency spacing. A most general theory of stage I has been worked out by Zweig and Shera (1995). They developed the notion of 'coherent reflection', i.e., reflection occurring by random irregularities, they described the criteria for this phenomenon to occur and showed that it can account for many properties of SOAEs. This concept was applied later, amongst others, by Talmadge et al. (1998), and further refined by Shera (2003). The original derivation was made for a one-dimensional (long-wave) model; in a recent paper the derivation has been extended to a two-dimensional model, a model that incorporates long as well as short waves (Shera et al. 2005). In the present paper it is tacitly understood that the backward-traveling wave-arising from coherent reflection-is governed by the same physical principles as the forward-traveling wave. In other words, we do not, at this moment, consider the possible influence of a compression wave.

In the Nuttall et al. (2004) paper it was reported that, before the spontaneous BM oscillation was noted as well as after it had subsided, the measured $\mathrm{BM}$ frequency response showed a number of fairly narrow peaks, the largest one corresponding in frequency with the oscillation. In that experiment a wide-band acoustical stimulus was used to determine the frequency response. When the level of the stimulus was increased, these peaks diminished in height, in other words, the peaks showed, more or less, the normally observed amplitude compression. The simplest explanation of our observations would involve assuming a single place of reflection along the length of the BM in the cochlea. We found, however, that the pattern of peaks is too extensive to be explained in this way. In the present paper the question is asked whether the response pattern found in that animal can be explained on the basis of 'coherent reflection' occurring in a three-dimensional model of the cochlea. The answer to this question is found to be affirmative. It is also shown that in the model used all the extra peaks are really due to superposition of the primary wave and a reverse propagating wave, which is reflected by the stapes; this finding confirms the validity of the underlying principles. Furthermore, a deeper study is dedicated to the variations of the peak amplitudes with stimulus intensity, and a good deal of correspondence with the data is found. Finally, the comparison of experiment and theory leads to a minor refinement regarding the actual requirements for coherent reflection in the cochlea.

\section{COHERENT REFLECTION}

The principle of coherent reflection can be explained in the simplest possible form as follows. All along the propagation course of waves in the cochlea there can occur reflections that are due to irregularities of the anatomy and the physiology. Each irregularity sends off two wavelets, one of these can be designated as 'reflected', the other one as 'scattered'. Where reflected wavelets from different locations add in a constructive way they can give rise to a sizeable reflected wave traveling toward the stapes.

To understand the principle better, consider a single-frequency wave. As it travels along the length of the basilar membrane (BM), its propagation velocity varies from location to location, and its wavelength and wave number vary likewise. Take the location where the wavelength is, say, $2 \mathrm{~mm}$. Around that location two irregularities $1 \mathrm{~mm}$ apart could give rise to a reflected wavelet (two times $\pi$ radians of phase difference, one time from forward, and one time from backward propagation). At a location farther from the stapes the wavelength may have decreased to $1 \mathrm{~mm}$, the critical distance of irregularities will then be $0.5 \mathrm{~mm}$, etc., etc. Therefore, the average density of irregularities that might give rise to a reflected wave will vary from location to location. For a sizable reflected wave to occur, it is necessary that the irregularities have a more or less random distribution over the length of the BM. More precisely, all wavelengths that can be involved in local wave propagation should be contained in them. This simplified reasoning explains why irregularities have to be truly 'irregular' in order to be able to cause reflection. The second requirement for appreciable reflection to occur concerns amplification. In a region where the primary wave is amplified, the reflected wave will be amplified, too. Therefore, as regards the ultimate amplitude of the reflected wave, this varies roughly as the square of the amplification factor. Therefore, coherent reflection can only occur a) in a region where there is appreciable amplification, and b) where there is much less am- 
plification in flanking regions. These are the main requirements for coherent reflection. More quantitative details are found in Zweig and Shera (1995), Talmadge et al. (1998) and Shera (2003), Shera et al. (2005). In what follows we will see the underlying principles at work.

\section{DATA PROCESSING TECHNIQUES - THE INVERSE SOLUTION}

The cochlea is a frequency-dependent nonlinear system. One way to stimulate such a structure and to extract relevant information about its functioning has been described by de Boer (1997). In order to apply the underlying theorem (called the EQ-NL theorem), it is compulsory to use a wide-band random-noise signal as the acoustical stimulus and to measure the input-output cross-correlation function (input is the velocity of the stapes, output the velocity of the BM, both being functions of time). The value of that stimulation method and the analysis it gives rise to will become evident shortly. In further interpretation of the results use is made of 'scaling', i.e., the property that the response pattern in the cochlea shifts when the frequency is varied. Hence we start with the Fourier Transform of the measured cross-correlation function, and consider this as a frequency response. That response is a (complex) function of frequency $f$ valid for one location $x$ (measured along the length of the BM), and it can be converted to a function of $x$. In the conversion the frequency $f$ is assumed to vary as an exponential function of $x$ (see for more details de Boer and Nuttall 1999). The resulting spatial response is valid for one frequency (usually the best frequency, $\mathrm{BF}$, of the location of measurement is chosen for this frequency). The spatial response, which is a complex function of $x$, will be referred to as a 'cochlear pattern'.

Next, a model of the cochlea is constructed. In view of the aforementioned EQ-NL theorem this model is a linear model. We have almost always used a model of which the geometry is constant over the full length of the model. To incorporate 'short' waves is essential for physical-mathematical reasons (de Boer 1979; Lighthill 1981). Therefore, the model has been made three-dimensional so that it can accommodate 'long' as well as 'short' waves - a threedimensional model (in which the $\mathrm{BM}$ is narrower than the width and the fluid displaced by the BM can thus move in three directions) is more realistic than a two-dimensional model. A set of convenient solution methods for this model has been published by de Boer (1998). Given a certain cochlear pattern, derived from a physiological experiment as described above, we can apply the 'inverse' solution method. With that method the BM impedance - which again is a function of $x$ - is determined in such a way that a cochlear model with this impedance produces a response that is identical to, or very similar to, the measured response (the cochlear pattern). This process is straightforward in a linear system. However, the cochlea is nonlinear. The earlier mentioned EQ-NL theorem states that the so-computed response of the model - which is linear - corresponds to the response of the original - nonlinear - cochlea, provided the latter is taken in the form of, or derived from, an input-output cross-correlation function for a wide-band stimulus. The same is true for all levels of stimulation (and thus for all degrees of average saturation of outer hair cells). This set of properties illustrates the value of the specific stimulation method with wide-band noise for a nonlinear system like the cochlea, and it is for this reason that we always have used this type of stimulation (see, for instance, de Boer and Nuttall 2000). It should be noted in passing that all parameters derived from the cochlear response, including the BM impedance, are to be regarded as averages over the excursions in time of the relevant signals (for instance, the BM displacement or the BM velocity or the pressure in the fluid).

\section{A VIEW AT DATA FROM THE ANIMAL WITH A SPONTANEOUS BM OSCILLATION}

In the Nuttall et al. (2004) paper on a spontaneous BM oscillation a few amplitude spectra were presented that were obtained with the wide-band noise stimulation method outlined in the preceding section (Fig. 8c in that paper, for details about the experiments we refer to the paper cited). For the present study the same frequency spectra have been heavily smoothed (by windowing the associated crosscorrelation functions in the time domain) and one result is shown here as Figure 1. Because of the smoothing the individual peaks are clearly visible although somewhat widened - in this figure. The stimulus signal was a two-octave wide band of pseudorandom noise, with a period of $\sim 20 \mathrm{~ms}$, presented continuously, and the mechanical response, the velocity, of the basilar membrane (BM) was averaged with the same period. Therefore, the spectral components were very close together ( $\sim 50 \mathrm{~Hz}$ spacing); too close to have them resolved in this figure. All spectra shown were normalized with respect to a fixed level, hence at low frequencies, where the cochlea is linear, responses for stimuli $10 \mathrm{~dB}$ apart show up as $10 \mathrm{~dB}$ apart. Thick and thin lines show the response spectra (amplitudes only) for various levels of the 


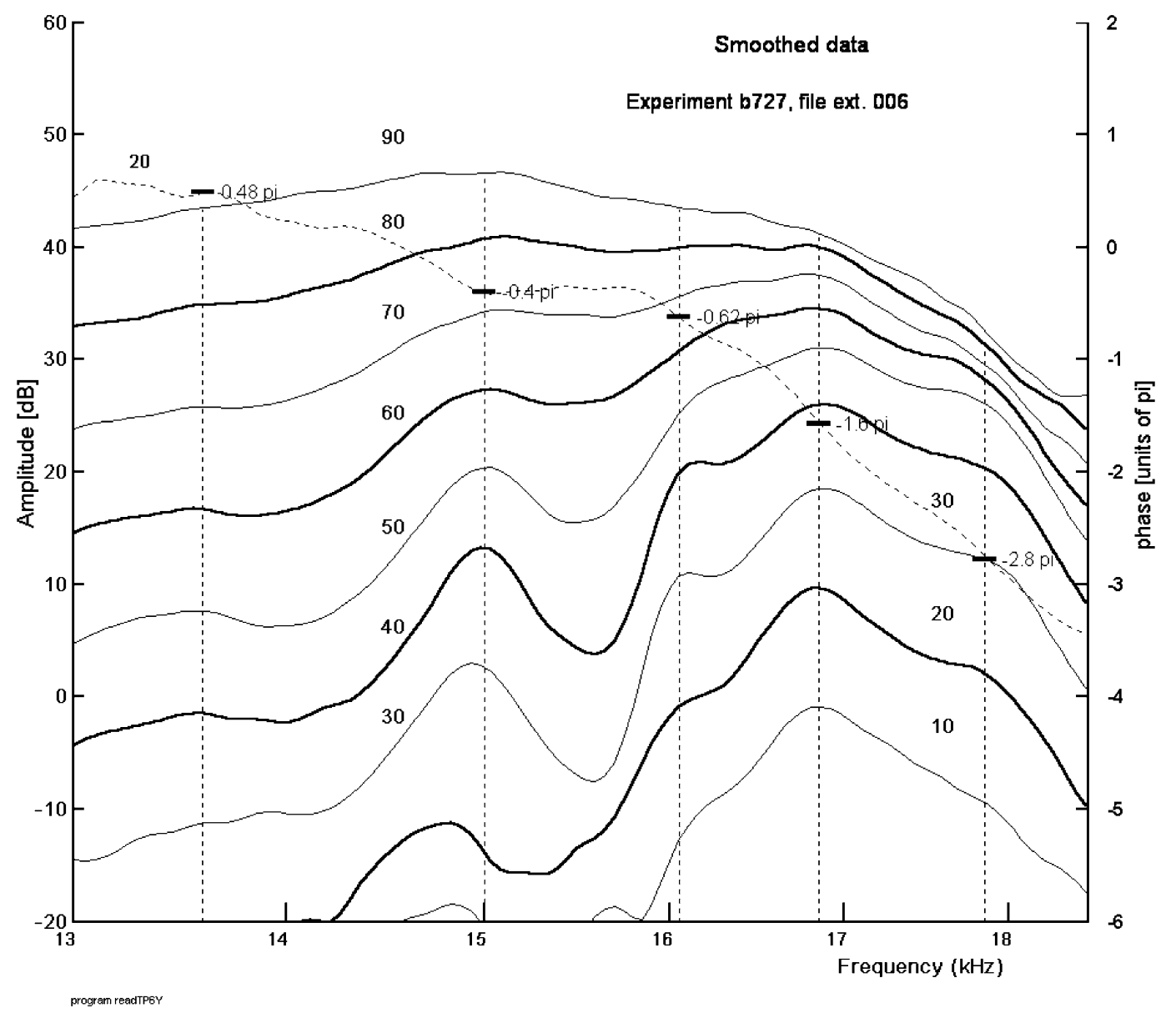

FIG. 1. Heavily smoothed frequency responses (BM velocity) of the animal with a spontaneous BM oscillation, experiment code: b727, equivalent to 24727 . Responses were measured with pseudorandom noise with a bandwidth of two octaves. Labels indicate level of the noise in dB SPL per octave (thick and thin lines alternate). Note the five peaks, marked by vertical dotted lines; all are dependent - in width and size, and somewhat in frequency - upon

stimulus intensity, in steps of $10 \mathrm{~dB}$. The labels indicate the SPL of one octave of the noise. The frequency scale of the figure encompasses less than one octave. The stimulus levels range from $10 \mathrm{~dB}$ to $90 \mathrm{~dB}$ SPL per octave, from a low value where the cochlea may be considered as linear to values where the cochlea is heavily driven into nonlinearity.

Nearly all of these curves show a number of amplitude peaks, in the figure they are connected by thin vertical dotted lines. The peak around $15 \mathrm{kHz}$ is the biggest and corresponds to the 'spontaneous oscillation' that was observed in this animal. Note that the size of all these peaks depends on stimulus level, the peaks disappear above $80 \mathrm{~dB}$ SPL per octave. We think it is important to note that the bandwidth of all peaks is noticeably smaller than that of the 'main' response peak (which can be identified to occur around $17 \mathrm{kHz}$ - the 'best frequency'). As said earlier, we did not find it possible to explain the level. The $15-\mathrm{kHz}$ peak is what remained of the spontaneous oscillation. On the ordinate scale, $0 \mathrm{~dB}$ corresponds to a velocity of $8.8910^{-4} \mathrm{~mm} . \mathrm{s}$, taken over a bandwidth of $50 \mathrm{~Hz}$. The thin dashed curve is the phase response at $20 \mathrm{~dB}$ per octave, the scale appears on the right. Note: responses have not been corrected for the stapes response.

pattern of peaks by the assumption that there is a single place in the cochlea (in the region tuned to 15 $\mathrm{kHz}$ ), which causes appreciable reflection. Accordingly, an intriguing question is whether the theory of coherent reflection applied to a three-dimensional model is able to explain:

a) the presence of these local peaks, and the possibility of a spontaneous oscillation,

b) the fact that these peaks are narrower than the 'main' response peak of the cochlea at this location, and

c) the variations of the amplitudes of these peaks when stimulus level is varied.

That theory requires the cochlea to have, along the length of the BM, a more or less random pattern of irregularities in structure or functioning. Although it is known that the guinea-pig cochlea is relatively regular in its structure (Wright 1984; Lonsbury- 
Martin et al. 1988) we believe that the particular animal studied (24727) might have been an exception (Nuttall et al. 2004).

\section{SETTING UP THE STAGE}

In the physiological experiment of which Figure 1 shows the spectra, insufficient data were taken to allow us to construct a suitable mechanical model for that particular animal. We have therefore considered another animal (code number 19922), its measured response to a wide-band stimulus signal and its derived BM impedance function. And we will modify the model associated with the latter animal in order to incorporate coherent reflection and to demonstrate properties associated with it. For low stimulus levels, the BM impedance shows 'activity', that is, over a certain segment on the stapes side of the response peak, the real part of the impedance is negative. In terms of the dynamics of the system this means that in that segment the power of the cochlear waves will be increased. It is generally assumed that outer hair cells - OHCs - are involved in this process of 'cochlear amplification'. It is in this 'active' segment where we can expect coherent reflection to occur (see Coherent Reflection Section).

Figure 2 shows a cochlear-pattern response (amplitude and phase) of animal 19922 in the upper panel and the BM impedance of the same animal in the lower panel, both depicted as functions of location $x$. The acoustical stimulus - wide-band noise, with a spectrum reaching from $125 \mathrm{~Hz}$ to $24 \mathrm{kHz}-$ had a low level: $20 \mathrm{~dB}$ SPL per octave. The sample rate was $208 \mathrm{kHz}$, the number of samples in the circular buffer 4096 so that the period was $19.696 \mathrm{~ms}$ (rounded off to $20 \mathrm{~ms}$ ). In this case the measured $\mathrm{BM}$ velocity was normalized by the velocity of the stapes for the same stimulus, as we have usually done in our analytical work. The thick amplitude curve in the upper panel shows the amplitude of the normalized BM velocity; the thin phase curve shows the corresponding phase. The BM impedance function, computed with the 'inverse method', is shown in the

$B M$ response, exper. 19922

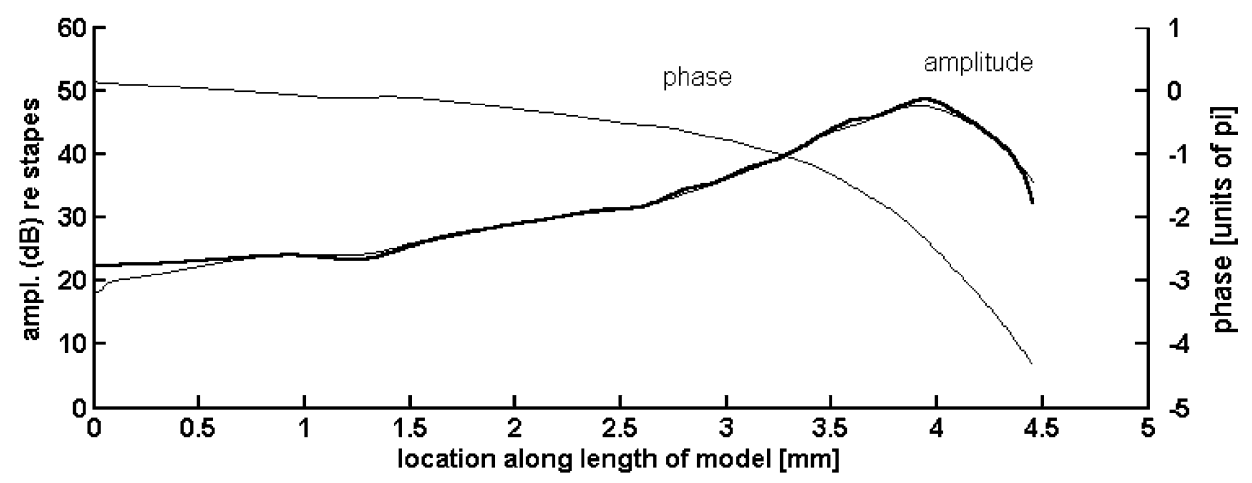

BM impedance

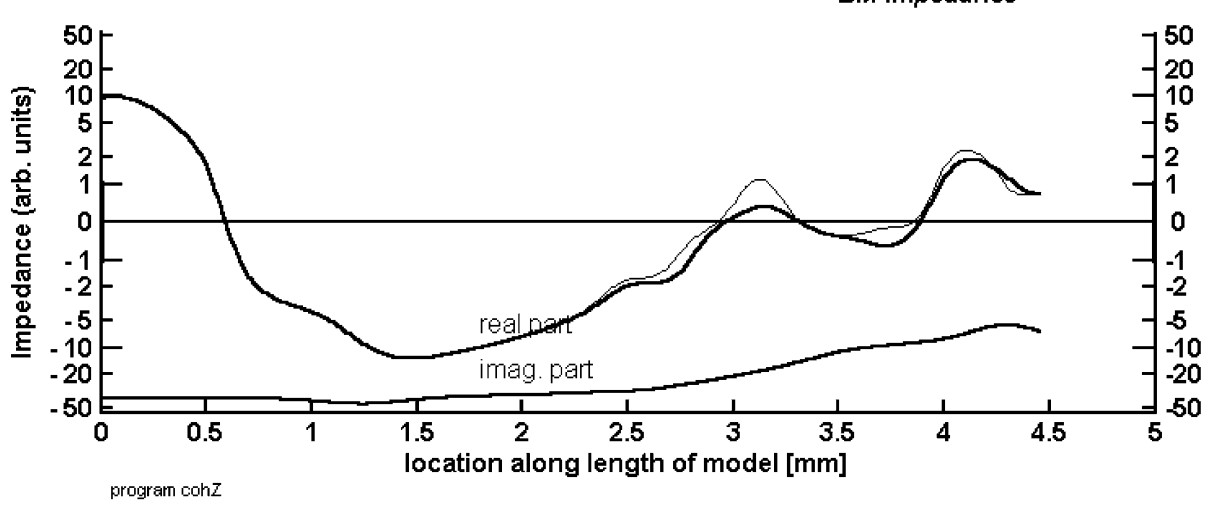

FIG. 2. Upper panel, BM velocity, converted from the frequency to the location $(x)$ domain, amplitude and phase. Data from another animal, code 19922. Location $x=0$ corresponds to the stapes. Thick amplitude curve: original BM velocity response, normalized with respect to the stapes response. Thin amplitude curve: BM velocity response after resynthesis (see text). Phase curve: phase of original
BM velocity, phase scale on the right. Lower panel: BM impedance recovered with inverse solution, real and imaginary parts. The ordinate scale is nonlinear. Thick curves: BM impedance derived from original response. These curves are valid for the frequency 16.5 $\mathrm{kHz}$. Thin curve: real part after random corrugations have been applied. This curve is also valid for $16.5 \mathrm{kHz}$. 
lower panel (real and imaginary parts, thick curves). When that impedance is inserted in a model, and the response of that model computed, the resulting response is called the 'resynthesized' response; the amplitude of this response is shown by the thin amplitude curve in the upper panel. The resynthesized response appears as somewhat smoother, this is due to the smoothing that the BM impedance function has undergone. The response and impedance functions shown in Figure 2 are valid for one frequency, in this case, $16.5 \mathrm{kHz}$, the best frequency at the location on the BM from which the measurements in animal 19922 were made.

Next, we introduce random variations of a limited size to the impedance. In that way we create a basilar membrane with 'irregularities' in its mechanics. The impedance function is perturbed, by applying a certain pattern of random variations to it (we will often refer to these variations as 'corrugations', the reason will become clear shortly). As a matter of fact, the random variations are only bestowed on the real part of the impedance. This is done because we expect that impedance variations will mainly arise from variations in the functioning of outer hair cells, and these cells have, in the relative sense, the greatest effect on the real part of the BM impedance and on cochlear amplification (see, for instance, de Boer and Nuttall 2000). For reasons of clarity the variations have been made largest around location $x=3.5$ (mm). The spectrum (actually, the wave number spectrum) of the variation function has been chosen to be a low-pass spectrum, so that impedance variations faster than 6.6 per $\mathrm{mm}$ will not occur. By limiting the wave number spectrum in this way we ensure the impedance variations to be reasonably smooth. The perturbed impedance is shown by thin curves in the lower panel of Figure 2. Let it be recalled that all functions shown in Figure 2 are functions of $x$ valid for one frequency.

The next step towards deriving a frequency response involves computing the impedance function in the $x$-domain for a large range of frequencies. Hereby the basic impedance function is shifted in place (remember, the cochlea 'scales' frequency $f$ and location $x$ ) but the imposed impedance variations are taken as invariant and fixed in space. Figure 3

$B M$ response, exper. 19922

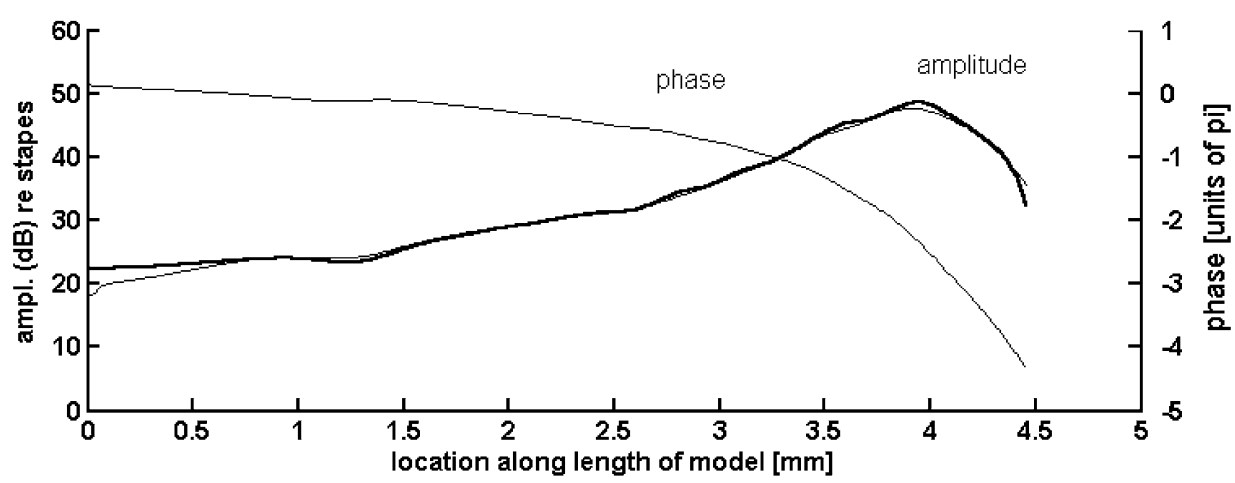

BM impedance

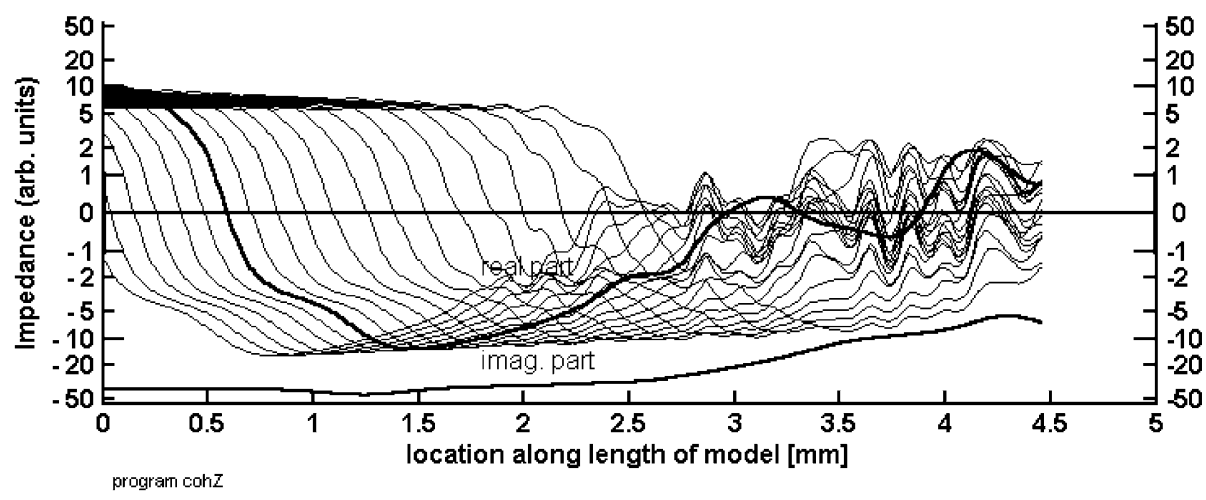

FIG. 3. BM response (upper panel) and BM impedance (lower panel), layout as in Figure 2. Thick lines in lower panel: impedance for $16.5 \mathrm{kHz}$ as shown in Figure 2. Thin lines: the perturbed impedance (real part) for a range of 20 frequencies, from 9.95 to $19.95 \mathrm{kHz}$ in steps of $500 \mathrm{~Hz}$. The basic impedance function scales with frequency: For lower frequencies the impedance curve lies more to the right. Because of the nonlinear ordinate scale the corrugations are best visible where the real part of the impedance is around zero. The place-fixed character of the corrugations is obvious. 
illustrates the procedure. The thick curves in this figure are the same as in Figure 2. In the lower panel the real part of the impedance function is shown, by way of thin curves, for a number of frequencies, from 9.95 to $19.95 \mathrm{kHz}$ in steps of $500 \mathrm{~Hz}$. For lower frequencies the basic impedance curves lie more to the right, for higher frequencies more to the left. It should now be evident why the impedance variations can be called 'corrugations', and the average size of the corrugations can be judged from the figure (note the nonlinear ordinate scale). In relation to the magnitude of the impedance the corrugations are of the order of $5 \%$. In the actual computation, the perturbations are applied to the impedance over the range of frequencies from 9.95 to $19.97 \mathrm{kHz}$ but in much smaller steps, namely, 197 steps of $50.78 \mathrm{~Hz}$.

\section{THE RESULTING PERTURBED FREQUENCY RESPONSE}

For each of the so-obtained 197 shifted and perturbed impedance functions resynthesis is done, resulting in 197 model-response functions, all complex functions of $x$. The values of all of these functions taken at one particular value $x_{0}$ of $x$ are stored; they constitute the model-predicted frequency response for that location, from 9.95 to $19.97 \mathrm{kHz}$. For $x_{0}$ we take in this case the location corresponding to the best frequency, $16.5 \mathrm{kHz}$, which is $4.0 \mathrm{~mm}$, this being the value that we have used earlier for transforming the measured response from the frequency $(f)$ to the location $(x)$ domain. The result of the entire procedure is shown by the frequency response in Figure 4. All values are referred to the stapes velocity at the same level of stimulation (20 dB SPL per octave). The original (resynthesized) amplitude frequency response is traced with thin lines, the amplitude response with corrugations on the BM with thicker lines. The changes in the phase response see the undulations - are minimal. In the amplitude response a number of more or less equidistant peaks are seen. The pattern of these peaks is similar to that in the data from Figure 1. As the finely dashed lines and arrows indicate, several of these peaks correspond roughly to differences of $\pi$ radians in the phase (incident and doubly reflected waves then

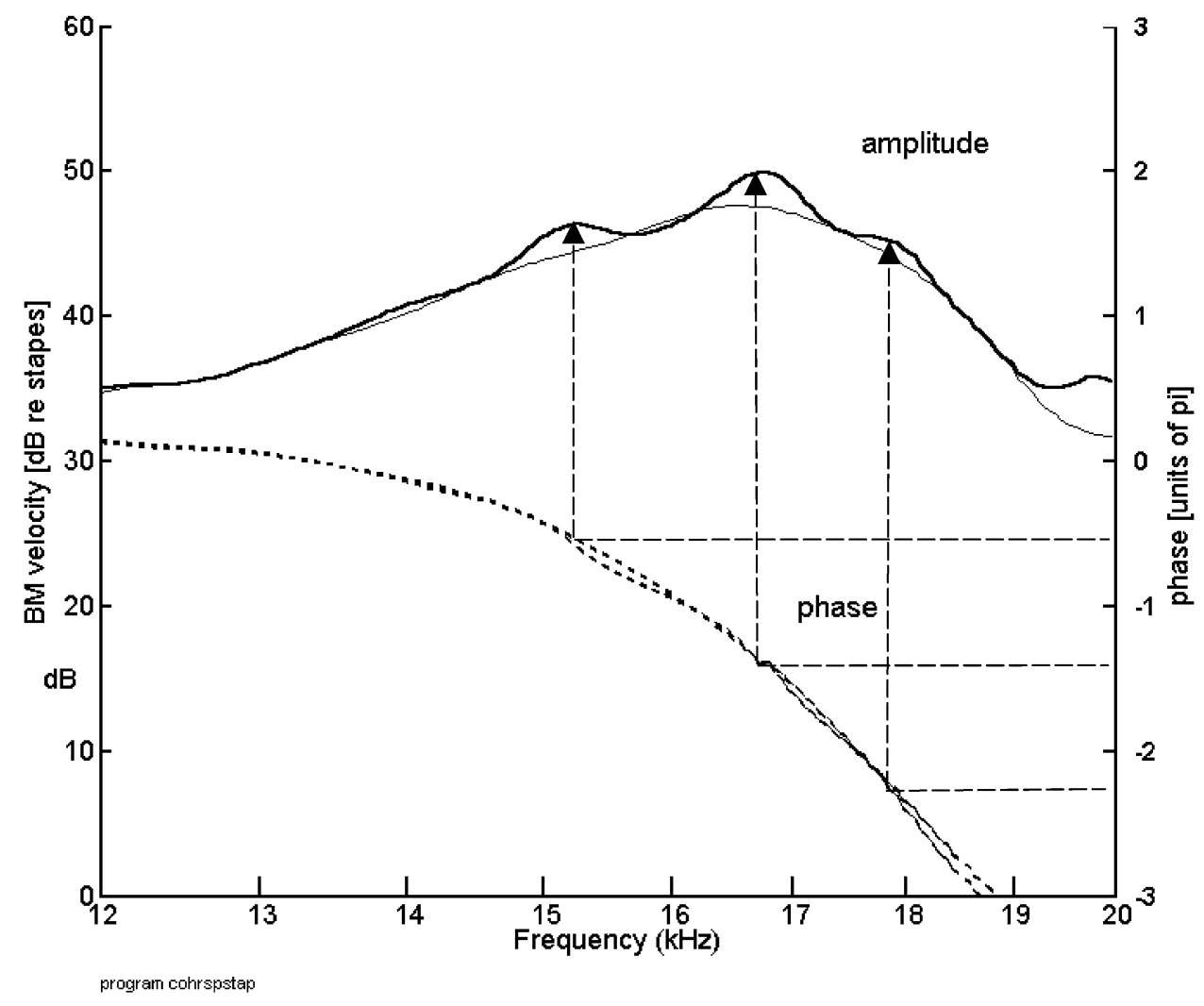

FIG. 4. Frequency response of the three-dimensional cochlear model with corrugations applied to the BM impedance function. Solid lines, amplitude in $\mathrm{dB}$, finely dashed lines, phase (see ordinate scale on the right). Thin curves: no corrugations; thicker curves: corrugations applied. In the latter case the response amplitude shows a series of more or less equidistant narrow peaks. Their average distance is comparable to that of the extra peaks in Figure 1. The finely dotted lines and arrows indicate that the phase difference between the peaks is not far from $\pi$ radians. 
have a phase difference of $2 \pi$ radians). We should not take that too literally, however, because (coherent) reflection - being based on random perturbations - introduces unpredictable phase variations. We have repeated the procedure several times, with different random functions for the corrugations. The pattern of peaks is different in each case but the average spacing remains about the same.

There is a fair degree of correspondence between the data we obtained from the animal with a spontaneous oscillation of the BM, Figure 1 (lower stimulus levels), and the computed response of a model provided with mechanical corrugations of the BM, Figure 4. The simulated response shows a series of isolated peaks that have an approximately constant frequency difference and are narrower than the main response peak around the best frequency $(16.5 \mathrm{kHz})$. With this result we have answered questions (a) and (b) of A View at Data from the Animal with a Spontaneous BM Oscillation Section in the affirmative: Coherent reflection can be provoked from a random corrugation pattern and gives rise to characteristic disturbances in the model response which are similar to those observed in a specific animal, property (a). Further, coherent reflection results in peaks that are narrower than the main response peak (property b). It is not difficult to see that, when we slightly increase the size of the corrugations, a condition can arise that gives rise to spontaneous oscillation (the second part of property a). We cannot simulate that with our computations, of course, because our model is linear and should remain stable. Finally, we want to stress that most of the effects of coherent reflection are visible in the region of the major response peak, that is, in the region where wave propagation in the cochlea occurs according to the short-wave model. Note that the original derivation for coherent reflection was carried out on a long-wave model. Apparently, the principle of coherent reflection is a universal one (cf. Shera et al. 2005).

The next step is to prove that the peaks are really due to interference of two waves, the primary wave and the twice-reflected wave, and not to another mechanism. In the model used we can do that fairly easily. The model is formalized as a matrix equation,

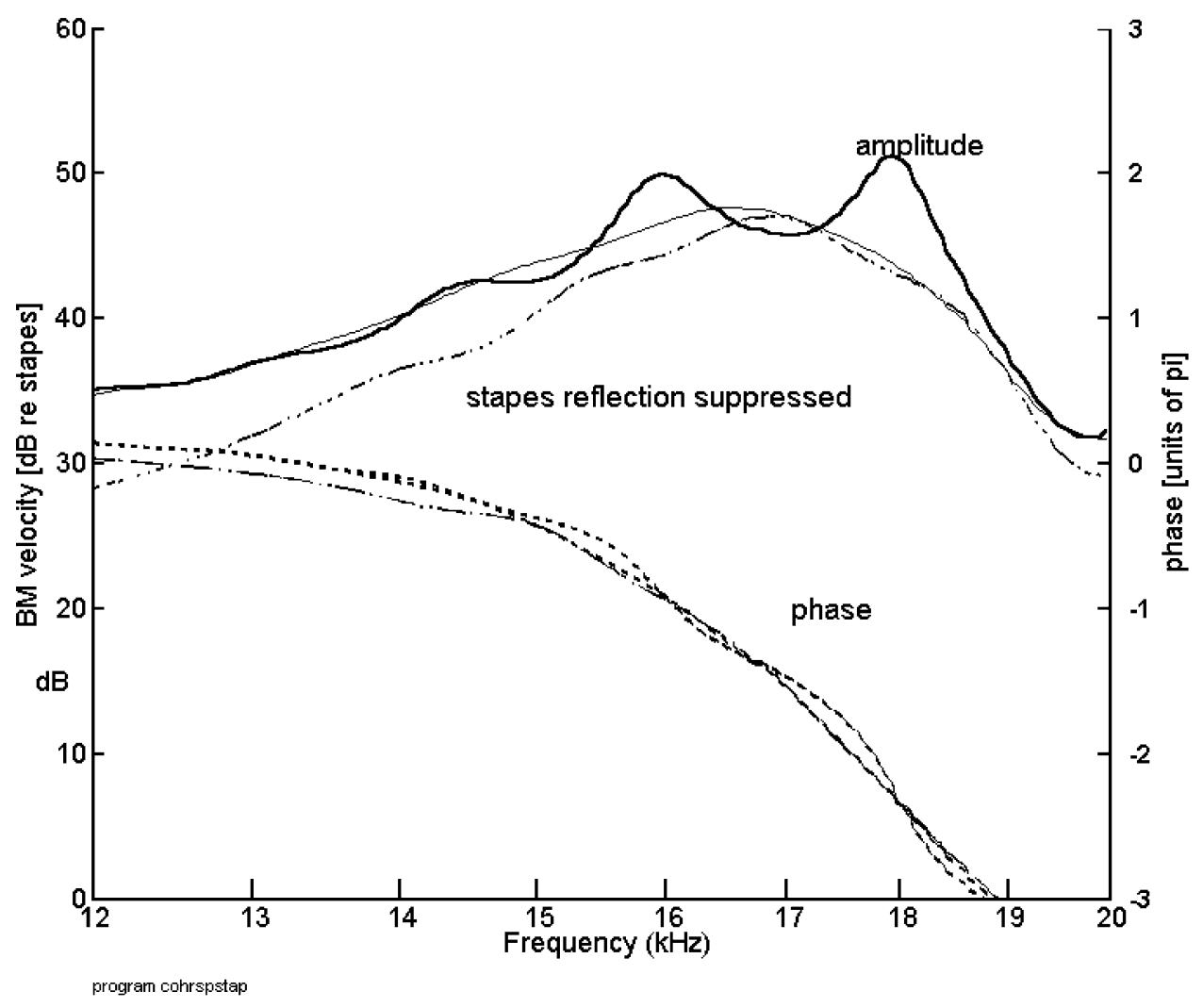

FIG. 5. As Figure 4, frequency response of cochlear model with a specific set of corrugations applied to the BM impedance function. The corrugations are different from those applied in Figure 4. The dash-dot lines show amplitude and phase computed in the same model, with the same corrugations, but with reflection from the stapes suppressed. Peaks are still visible but they are much smaller, and lie at different frequencies. Undulations in the phase have nearly disappeared. 
in each row of the matrix are condensed the mechanical properties of the basilar membrane at one location as well as those of the cochlear fluid all around it (cf. de Boer 1998). The unknown variable in the matrix equation is the pressure in the fluid very close to the (midline of the) BM. The first row of the matrix describes conditions at the stapes location, it normally expresses the fact that the stapes velocity is given and that the fluid has to follow. That row can simply be modified to mean that the stapes is driven by a velocity source via an impedance. For the parameter we take the 'characteristic impedance' at the stapes location, or its complex conjugate (de Boer 1984); in this way we artificially ensure that a minimal amount of reflection takes place at the stapes. Figure 5 shows a typical result. Firstly, it shows a computed frequency response just as Figure 4, but with a different set of corrugations. Therefore, the locations and heights of the peaks and valleys are different from those in Figure 4. Secondly, the computation is repeated with the same impedance corrugations but with the stapes driven by the appropriate impedance so as to suppress reflection at that location. The result is shown by the dash-dot lines. Apart from a general decrease of about $6 \mathrm{~dB}$ (the characteristic impedance approximately matches the input impedance of the model) the peaks and valleys have diminished considerably in magnitude. We may conclude that the original peaks and valleys indeed result from the addition of two waves of which one has been reflected two times. The remaining undulations with stapes reflection reduced are due to imperfect elimination of reflection by the stapes and 'coherent scattering' of the primary wave, a necessary by-product of coherent reflection.

\section{EFFECTS OF STIMULUS INTENSITY}

The effects illustrated by Figures 4 and 5 are derived from a response function obtained from animal 19922 at a very low level of stimulus intensity (20 dB SPL per octave). At higher levels that animal showed the usual variations of response and impedance (de Boer and Nuttall 2000). In the context of the EQ-NL theorem, the obtained response functions (all derived from input-output cross-correlation functions as described in Data Processing Techniques - The Inverse Solution Section) have a specific meaning. We repeat: When from a measured response the corresponding BM impedance function is derived and resynthesis applied, the resulting model re-

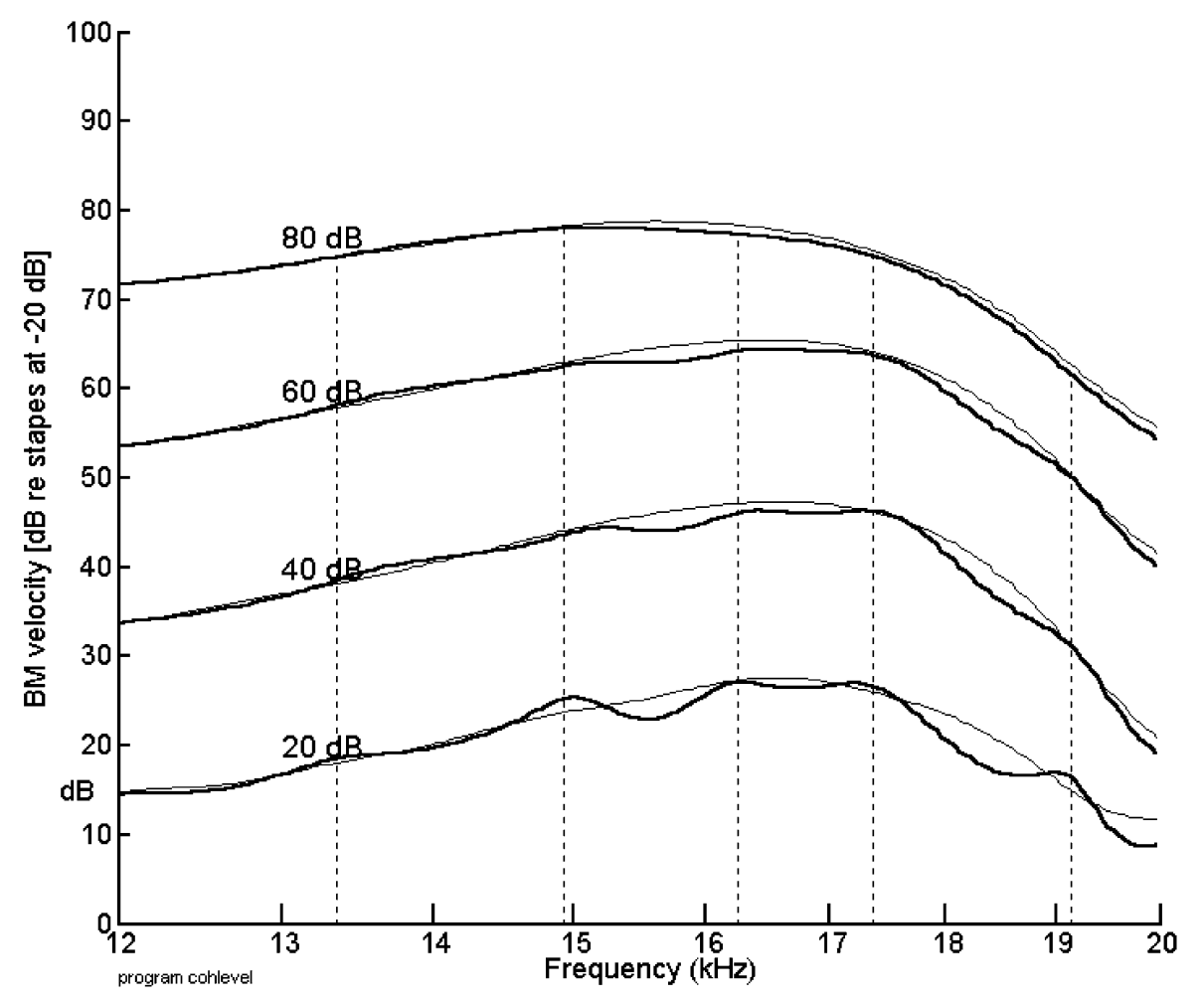

FIG. 6. Computed response curves (amplitudes) for various stimulus levels (20 to $80 \mathrm{~dB}$ per octave in steps of 20). Original frequency-response data from animal '19922'. For all stimulus levels the same pattern of corrugations was used (different from the one in previous figures). The vertical dotted lines indicate the individual peaks. With increasing stimulus level the amplitude of the peaks decreases in about the same way as in the experimental data of Figure 1. 
sponse is identical to or very close to the measured response function. Note that the resynthesized response is from a linear model whereas the measured response is from a nonlinear system. This correspondence holds true for all degrees of saturation, i.e., for all stimulus levels.

This reasoning leads to the next stage in our analysis: We should do the simulation procedure with the same artificial corrugations and apply it to data obtained from animal 19922 over a range of stimulus levels. For the preparation of the next figure we have taken the following steps. Beforehand we fixed a pattern of impedance corrugations as described above. Response functions - all measured via the cross-correlation technique - were taken from the same animal, 19922, for a series of stimulus levels (from 20 to $80 \mathrm{~dB}$ SPL per octave). Each of these frequency responses was converted to the $x$-domain, and subjected to the inverse solution to find the associated BM impedance function. Next, following Setting Up the Stage Section, we converted each impedance function to the range of frequencies from 9.95 to $19.97 \mathrm{kHz}$, and imposed the corrugations upon it. Let it be repeated that we have used the same pattern of corrugations at every stimulus level. Finally, we computed the frequency response as in The Resulting Perturbed Frequency Response Section, for each of the stimulus levels. Plotting of the results is done in a different way than the one used in Figures 4 and 5. All curves are now plotted with respect to the stapes response at a fixed level (in this case $-20 \mathrm{~dB}$ SPL per octave). In fact, this is similar to the way of plotting in Figure 1. Because of this normalization the amplitudes for low frequencies - where there is no amplitude compression - are $20 \mathrm{~dB}$ apart for every $20 \mathrm{~dB}$ of intensity difference.

Figure 6 shows a result of this procedure. To avoid cluttering the figure, we have plotted only results for 20, 40, 60 and $80 \mathrm{~dB}$ SPL per octave. The size of the typical, reflection-caused, peaks is seen to decrease with increasing level in approximately the same way as in the data of Figure 1. For the sake of completeness, we have repeated the procedure for data from the same animal using a different pattern of corrugations. The result is shown by Figure 7 . The distribution of peak frequencies is seen to be different from that in Figure 6, but the way in which the peak amplitudes decrease with increasing level is qualitatively the same. With these findings the question of item (c) in A View at Data from the Animal with a Spontaneous BM Oscillation Section is answered affirmatively.

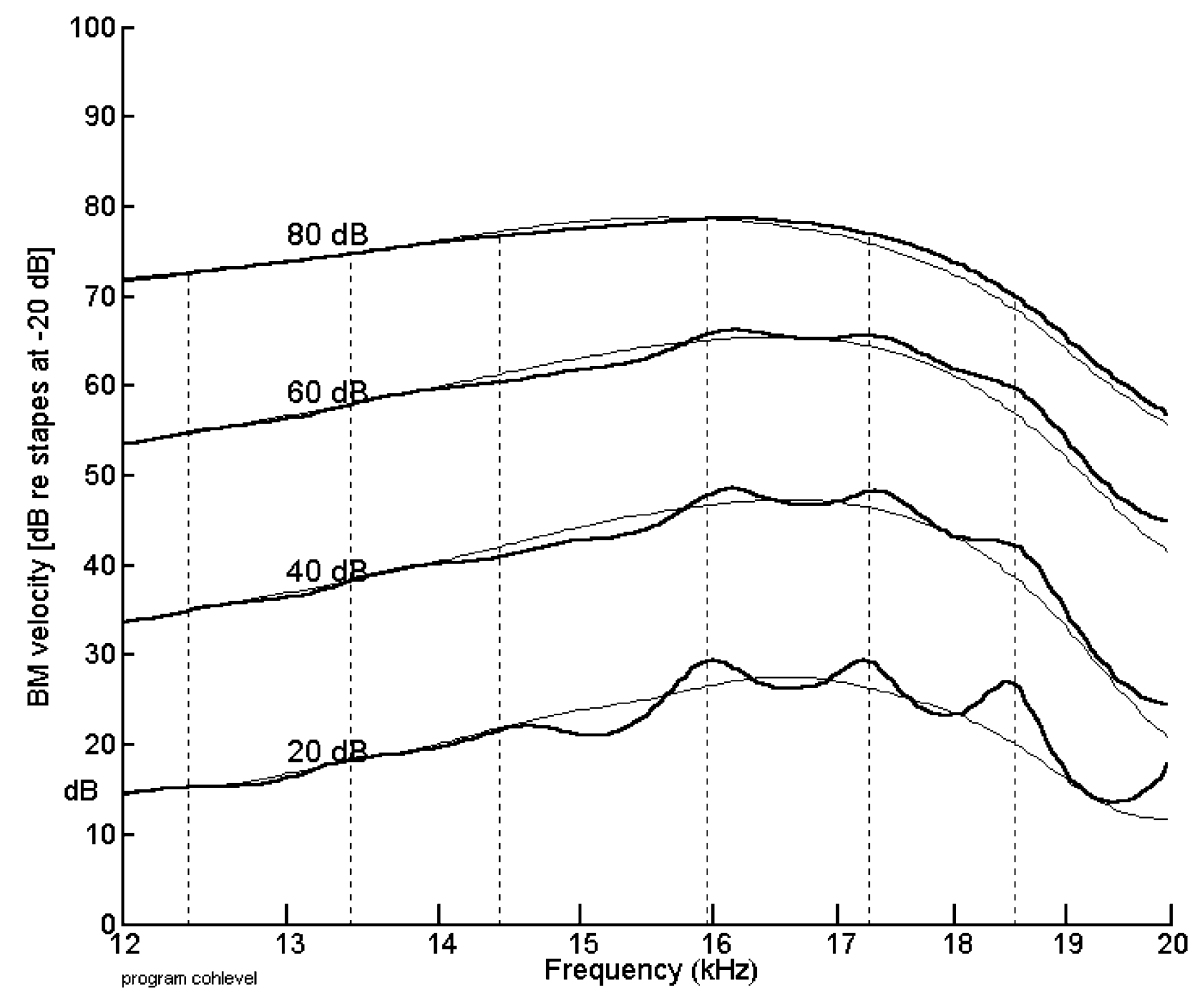

FIG. 7. Computed response curves (amplitudes) for various stimulus levels (20 to $80 \mathrm{~dB}$ per octave in steps of 20). Original frequency-response data from the same animal, corrugation pattern different. As a result the frequencies of the extra peaks are different from those in Figure 6, but the way in which the size of the peaks diminishes with increasing stimulus level is similar. Note the (unexplained) tendency of the peaks to move upward in frequency when the sound level increases. 


\section{SUMMARY AND COMMENTARY}

In this report we have shown that providing for coherent reflection in a three-dimensional model of the cochlea can give rise to characteristic disturbances in the response, extra peaks arising with a more or less constant frequency difference. These extra peaks are similar to those observed in a specific animal (24727), the one that has shown spontaneous oscillations of the BM. We also showed that the pattern of these computed peaks depends, as expected, on the choice of the random pattern of corrugations applied in the model computation. Furthermore, we demonstrated that the peaks are really due to the superposition of two waves, the primary wave set up by the stimulus, and a second wave that results from a reverse propagating wave which is due to 'coherent reflection' - being reflected by the stapes. It is easy to see that, when we would increase the size of the corrugations, we could approach a condition of spontaneous oscillation. In this way we have fully answered question (a) of A View at Data from the Animal with a Spontaneous BM Oscillation Section: Local peaks are explained and spontaneous oscillation is possible. Is it surprising that the extra peaks generated by coherent reflection are narrower than the main response peak (question b)? The answer is: No, not at all. The spacing of the peaks is governed by the intrinsic phase response, in particular, extra peaks can arise where the response phase changes by approximately $\pi$ radians, see Figure 4 . The phase range covered by the main response peak - which is the broad peak centered at $16.5 \mathrm{kHz}$ - is larger, hence the main peak can include several sub-peaks.

Finally, we applied the same computational technique, with the same pattern of corrugations, to data obtained at various levels of stimulation, and we found (Figs. 6 and 7) that with increasing stimulus level the sizes of the peaks diminish in approximately the same way as they do in the data of the original animal with the spontaneous oscillation (Fig. 1). In this way we also have answered question (c) of A View at Data from the Animal with a Spontaneous BM Oscillation Section.

An intriguing problem seems to be that in our computation results the frequencies of the extra peaks have a tendency to increase in frequency with stimulus level. Why this should be so and whether it is an intrinsic property of the system remains to be seen; if true, it is probably related to the small but systematic flattening of the phase response curves with increasing stimulus level. In the original experimental data of Figure 1 we see this feature in the peak at $15 \mathrm{kHz}$ but to a much lesser extent in the other peaks. Furthermore, the extra peaks in
Figures 6 and 7 do not clearly show the typical bandwidth widening that the main response peak demonstrates (see the thin lines). As explained earlier, the extra peaks have an intrinsic separation, which can be expected to depend only little on stimulus level because the phase variations with level are small. As an additional item it is stated that we did not intentionally attempt to simulate a cochlea that has the largest possibility of becoming unstable at $15 \mathrm{kHz}$. We chose to illustrate more general properties of wave travel and coherent reflection instead, and therefore concentrated on the region around $16.5 \mathrm{kHz}$. That is probably also the reason why the separation of the measured peaks in Figure 1 does not exactly correspond to that in the simulation figures.

A few further questions may be raised. In Coherent Reflection Section we noted that the amplitude of the reflected wave is quadratic in the amplification factor. The twice-reflected wave is amplified again before it adds to the incident wave, but this one is amplified as well, hence the size of the interference peak (expressed as a ratio of amplitudes) will also vary as the second power of the amplification. On this basis we might have expected the size of the peaks to diminish more rapidly with increasing stimulus level than we observe it in Figures 6 and 7. The answer to this paradox lies in the intricate relation between cochlear amplification and maximum amplitude of response. In an earlier paper (de Boer and Nuttall 2000) we have made a careful study of that relation. Assuming that variations of cochlear amplification were due to partial saturation of the 'active' mechanism, we could completely account for a) the observed variations of the maximum response amplitude as well as b) the variations in cochlear tuning. In the simplest terms: Compression of the maximal response amplitude is much larger than the variation occurring in cochlear amplification due to saturation (compare Patuzzi et al. 1989). In still different terms: 'amplification' should not be judged on the basis of maximum response. And translated to the present situation: The variations of cochlear amplification are relatively small, and therefore the effect of stimulus level on peak size is gradual.

What were the fundamental requirements for coherent reflection? An important one was amplification, which should be manifest over a restricted segment along the length of the BM (Zweig and Shera 1995). Since our procedure still works at high stimulus levels, where maximum response amplitude has diminished considerably, we conclude that the 'amplification requirement' should refer to 'cochlear amplification' and not to 'response amplitude' per se. Note, in this connection, that we have applied the corrugations to what we consider to be the compo- 
nent controlling cochlear amplification: the real component of the BM impedance.

The subject of reverse propagation of waves in the cochlea has recently received a new impulse, starting by observations of Ren (2004a, b), which raise the question whether 'compression waves' would be important. Our mathematical treatment has exclusively been based on anti-symmetrical waves in the cochlea (equal amplitudes but opposite signs of the fluid pressures in the two channels of the model). Physically, this means that in our model fluid waves of this type can freely propagate in the two directions, forward and reverse, and our solutions of the matrix equation have automatically included these waves. On the basis of our data we can justify the inclusion of reverse anti-symmetrical waves. Consider the phase curve depicted in Figure 1. The phase values at the intersections with the five vertical dotted lines are indicated. From the four phase intervals three are of the order of $\pi$ radians. For a reinforcement of the amplitude a phase difference of $2 \pi$ radians between the consistuent waves is necessary. If forward and reverse waves travel with the same speed, each has to contribute $\pi$ radians for a peak to occur as we have shown in Figure 4. It is impossible to explain our data with a compressions wave (in which the pressures are equal in amplitude and in polarity in the two channels) playing the part of the reverse wave - in that case the phase steps between the peaks would be $2 \pi$ instead of $\pi$ radians. A more precise description of the differences between compression waves and propagating waves in relation to experimental results will be given in a further report.

\section{ACKNOWLEDGMENT}

The authors are deeply indebted to Jiefu Zheng, who performed the physiological experiments with the greatest skill and acquired the animal data on which the theoretical work in this paper is based.

\section{REFERENCES}

DE Boer E. Short-wave world revisited: Resonance in a twodimensional cochlear model. Hear. Res. 1:253-281, 1979.
DE Boer E. Auditory Physics. Physical principles in hearing theory, part II. Phys. Rep. 105:141-226, 1984.

DE BoER E. Connecting frequency selectivity and nonlinearity for models of the cochlea. Audit. Neurosci. 3:377-388, 1997.

DE BOER E. A method for forward and inverse solutions of a 3-dimensional model of the cochlea. J. Acoust. Soc. Am. 103:3725-3728, 1998

DE BOER E, Nuttall AL. The "inverse problem" solved for a threedimensional model of the cochlea. III. Brushing-up the solution method. J. Acoust. Soc. Am. 105:3410-3420, 1999.

de Boer E, Nuttall AL. The mechanical waveform of the basilar membrane. III. Intensity effects. J. Acoust. Soc. Am. 107:14971507, 2000.

Hilger AW, Furness DN, Wilson JP. The possible relationship between transient evoked otoacoustic emissions and organ of Corti irregularities in the guinea pig. Hear. Res. 84:1-11, 1995.

KEMP DT. Stimulated acoustic emission from within the human auditory system. J. Acoust. Soc. Am. 64:1386-1391, 1978.

KEMP DT. Evidence of mechanical nonlinearity and frequency selective wave amplification in the cochlea. Arch. Otorhinolaryngol. 224:37-45, 1979 .

LightHill MJ. Energy flow in the cochlea. J. Fluid Mech. 106:149213,1981

Lonsbury-Martin BL, Martin GK, Probst R, Coats AC. Spontaneous otoacoustic emissions in a nonhuman primate. II. Cochlear anatomy. Hear. Res. 33:69-93, 1988.

Nuttall AL, Grosh K, Zheng J, de Boer E, Zou Y, Ren T. Spontaneous basilar membrane oscillation and otoacoustic emission at $15 \mathrm{kHz}$ in a guinea pig. J. Assoc. Res. Otolaryngol. 5:337-349, 2004.

Patuzzi RB, Yates GK, Johnstone BM. Outer hair cell receptor cur-rent and sensorineural hearing loss. Hear. Res. 42:47-72, 1989.

REN T. Propagation direction of the otoacoustic emission along the basilar membrane. Association for Research in Otolaryngology, Mid-Winter-Meeting Abstracts, 27: 343 (\#1011), 2004a.

REN T. Reverse propagation of sound in the gerbil cochlea. Nature Neurosci. 7:333-334, 2004b.

SHERA CA. Mammalian spontaneous otoacoustic emissions are amplitude-stabilized cochlear standing waves. J. Acoust. Soc. Am. 114:244-262, 2003.

Shera CA, Tubis, A, Talmadge, CL. Coherent reflection in a twodimensional cochlea: Short-wave versus long-wave scattering in the generation of reflection-source otoacoustic emissions. J. Acoust. Soc. Am. 118:287-313, 2005.

Talmadge C, Tubis A, Long GR, Piskorski P. Modeling otoacoustic emissions and hearing threshold fine structures. J. Acoust. Soc. Am. 104:1517-1543, 1998.

WRIGHT AA. Dimensions of the cochlear stereocilia in man and in guinea pig. Hear. Res. 13:89-98, 1984.

Zweig G, Shera CA. The origin of periodicity in the spectrum of evoked otoacoustic emissions. J. Acoust. Soc. Am. 98:20182047, 1995. 